TRANSACTIONS OF THE

AMERICAN MATHEMATICAL SOCIETY

Volume 363, Number 12, December 2011, Pages 6439-6452

S 0002-9947(2011)05263-3

Article electronically published on July 25, 2011

\title{
ANTISYMMETRIES OF THE CAR ALGEBRA
}

\author{
P. J. STACEY, \\ WITH AN APPENDIX BY JEFFREY L. BOERSEMA AND N. CHRISTOPHER PHILLIPS
}

\begin{abstract}
Let $A$ be a uniformly hyperfinite $C^{*}$-algebra with infinitely many $2 \times 2$ matrix factors. It is shown that, up to conjugacy, there is a unique antisymmetry (involutory $*$-antiautomorphism) of $A$.
\end{abstract}

\section{INTRODUCTION}

In a groundbreaking paper [1 in 1990, Blackadar produced an answer to the longstanding question of whether or not a period two automorphism (or symmetry) of an AF algebra possessed an AF fixed point algebra. He showed that, even in the case where the algebra in question was the uniformly hyperfinite $C^{*}$-algebra with infinitely many $2 \times 2$ matrix factors (the CAR algebra), the fixed point algebra may not be AF. He observed in a final section of the paper that "One might hope that a modification of the methods of this paper could provide a negative solution to the following well-known open question: is there an antisymmetry of the CAR algebra $A$ which is not locally representable?" Antisymmetries of a given $C^{*}$-algebra $A$ are in bijective correspondence with real $C^{*}$-algebras $R$ with complexification equal to $A$ (real forms of $A$ ): given an antisymmetry $\Phi$ the fixed point subalgebra of $\Phi \circ *$ is a real form of $A$ and, given a real form $R$ of $A$, the mapping $a+i b \mapsto a^{*}+i b^{*}$ for $a, b \in R$ is an antisymmetry. The present paper attacks Blackadar's question by analysing the real forms of the CAR algebra.

Since Blackadar's paper was written there have been major advances in the classification program for simple $C^{*}$-algebras. In particular, the work of Lin [12, [16, [14] has moved the program in the finite case past the state where an inductive limit structure is required. This provides a method for resolving Blackadar's question in the negative. The paper [23] shows that the framework used by Lin applies to the real algebra associated with an antisymmetry of $A$. It was shown by Blackadar in Theorem 8.4.2 of [1] that any such real algebra shares the same $K$-theory as the canonical real CAR algebra. Thus, the final step is now to show that Lin's methods carry through to the real case and enable the real algebras to be classified by their $K$-theory.

The $K$-theory for real $C^{*}$-algebras is considerably more complicated than in the complex case. In particular, the real counterpart to the Universal Coefficient Theorem of Rosenberg and Schochet [17, which was obtained by Boersema [3], uses a rather complicated theory of $C R T$-modules due to Bousfield 4. Rather than attacking the real counterpart to Lin's classification in full generality, the

Received by the editors October 25, 2007 and, in revised form, November 24, 2009.

2010 Mathematics Subject Classification. Primary 46L35.

(C)2011 American Mathematical Society Reverts to public domain 28 years from publication 
present paper restricts our attention to a simple situation which suffices to handle the problem under consideration.

\section{Absorbing extensions}

In this section it is shown that results of Dadarlat and Eilers [6] and of Lin [14, [15] are applicable in the real case. This is facilitated by the underlying work of Kasparov [1] covering the real as well as the complex case.

The presentation follows Section 2 of [6], so a representation of a real $C^{*}$-algebra $A$ will refer to a ${ }^{*}$-homomorphism from $A$ to the real $C^{*}$-algebra $\mathcal{L}_{B}(E)$ of adjointable $B$-linear operators on a real Hilbert $C^{*}$-module $E$, which is countably generated over a $\sigma$-unital real $C^{*}$-algebra $B$. $H_{B}$ denotes the canonical such module.

As in [6], two representations $\gamma: A \rightarrow \mathcal{L}_{B}(E)$ and $\gamma^{\prime}: A \rightarrow \mathcal{L}_{B}\left(E^{\prime}\right)$ are said to be approximately unitarily equivalent, written $\gamma \sim \gamma^{\prime}$, if there exists a sequence of unitaries $u_{n} \in \mathcal{L}_{B}\left(E^{\prime}, E\right)$ such that $\lim _{n \rightarrow \infty}\left\|\gamma(a)-u_{n} \gamma^{\prime}(A) u_{n}^{*}\right\|=0$ and $\gamma(a)-$ $u_{n} \gamma^{\prime}(a) u_{n}^{*} \in \mathcal{K}_{B}(E)$ for any $a \in A$. The definitions of $\mathcal{L}_{B}\left(E^{\prime}, E\right)$ and $\mathcal{K}_{B}(E)$ can be found in [11].

A unital representation $\gamma$ is said to be absorbing if $\gamma \oplus \sigma \sim \gamma$ for any unital representation $\sigma: A \rightarrow \mathcal{L}_{B}(F)$. A variant of this definition, introduced in [6], defines a unital representation $\gamma$ to be nuclearly absorbing if $\gamma \oplus \sigma \sim \gamma$ for any unital strictly nuclear representation $\sigma: A \rightarrow \mathcal{L}_{B}(F)$, where a completely positive contraction $\sigma: A \rightarrow \mathcal{L}_{B}(F)$ is said to be strictly nuclear if there exist integers $\left(n_{\lambda}\right)$ and generalised sequences $\psi_{\lambda}: A \rightarrow M_{n_{\lambda}}(\mathbb{R})$ and $\phi_{\lambda}: M_{n_{\lambda}}(\mathbb{R}) \rightarrow \mathcal{L}_{B}(F)$ of completely positive contractions with $\lim _{\lambda \rightarrow \infty} \phi_{\lambda} \psi_{\lambda}(a)=\sigma(a)$ in the strict topology for all $a \in A$. When $A$ is nuclear, as is the case when Proposition 3 is used in Section 4 of the present paper, all representations are strictly nuclear, so there is no need to distinguish between absorbing and nuclearly absorbing representations. Nevertheless, for possible later reference, the results in this section are stated as real variants of the results in 6 .

If $A$ is a separable real $C^{*}$-algebra, an admissible scalar representation $\theta: A \rightarrow$ $M\left(\mathcal{K}_{\mathbb{R}}(H) \otimes B\right)$ is a ${ }^{*}$-homomorphism which factors as

$$
A \stackrel{\theta^{\prime}}{\rightarrow} \mathcal{L}_{\mathbb{R}}(H) \stackrel{-\otimes 1}{\rightarrow} \mathcal{L}_{\mathbb{R}}(H) \otimes M(B) \rightarrow M\left(\mathcal{K}_{\mathbb{R}}(H) \otimes B\right),
$$

where $H$ is a separable real Hilbert space and $\theta^{\prime}$ is faithful and of infinite multiplicity. We first note the following real analogue of Proposition 2.18 of [6].

Proposition 1. Let $A$ be a unital separable real $C^{*}$-algebra and let $B$ be a $\sigma$-unital real $C^{*}$-algebra. If $\theta: A \rightarrow \mathcal{L}_{B}\left(H_{B}\right)$ is a unital admissible scalar representation, then $\theta$ is nuclearly absorbing.

Proof. The proof proceeds exactly as in Proposition 2.18 of [6], noting that the cited results from [11] are explicitly proved by Kasparov for the real as well as the complex case.

The proof of the real analogue of Theorem 2.22 of [6] cannot proceed exactly as in [6] because Lemma 2.20 in [6] is no longer true for real $C^{*}$-algebras. A counterexample is given by the algebra $\mathbb{H}$ of real quaternions, for which 1 is the only positive element of norm one and for which the only real state $\phi$ has

$$
\phi(a+b i+c j+d k)=a .
$$


However, Lemma 2.21 of [6] can nevertheless be adapted to the real case. As in [6], if $\iota: A \rightarrow B$ is a unital full embedding (i.e. has $\operatorname{span}(B \iota(a) B)$ dense in $B$ for all non-zero $a \in A)$, then $d_{\iota}: A \rightarrow M\left(\mathcal{K}_{\mathbb{R}}(H) \otimes B\right)$ is defined by $d_{\iota}(a)=1 \otimes \iota(a)$.

Lemma 2. Let $A$ be a unital separable real $C^{*}$-algebra, let $B$ be a unital real $C^{*}$-algebra and let $\iota: A \hookrightarrow B$ be a unital full embedding. Then, for any unital completely positive map $\phi: A \rightarrow M_{n}(\mathbb{R}) \subset M_{n}(B)=\mathcal{L}_{B}\left(B^{n}\right)$ there is a bounded sequence $v_{i} \in \mathcal{K}_{B}\left(B^{n}, H_{B}\right)$ such that $\lim _{i \rightarrow \infty}\left\|\phi(a)-v_{i}^{*}(1 \otimes \iota(a)) v_{i}\right\|=0$ for all $a \in A$ and $\lim _{i \rightarrow \infty}\left\|v_{i}^{*} \xi\right\|=0$ for all $\xi \in H_{B}$.

Proof. Fix a finite set $\mathcal{F} \subset A$ and $\epsilon>0$. As in [6] it suffices to take $n=1$ and to find $m$ and an isometry $V \in \mathcal{L}_{B}\left(B, B^{m}\right)$ with $\left\|\phi(a) 1_{B}-V^{*}\left(1_{m} \otimes \iota(a)\right) V\right\|<\epsilon$. Let $\Psi_{A}$ be the involutory antiautomorphism of $A \otimes \mathbb{C}$ associated with $A$, let $\Psi_{B}$ be similarly associated with $B$ and note that $\Psi_{B} \circ \iota^{\mathbb{C}}=\iota^{\mathbb{C}} \circ \Psi_{A}$, where $\iota^{\mathbb{C}}$ is the complexification of $\iota$.

Applying the proof of Lemma 2.21 of [6] to $\iota^{\mathbb{C}}$, to the complexification $\phi^{\mathbb{C}}$ : $A \otimes \mathbb{C} \rightarrow \mathbb{C}$ of $\phi$ and to the finite set $\mathcal{F} \cup \Psi_{A}(\mathcal{F})=\mathcal{F} \cup \mathcal{F}^{*} \subset A$ produces $m, k$ and $v_{1}, \ldots, v_{m k} \in B \otimes \mathbb{C}$ with $\left\|\phi(a) 1_{B}-\sum_{i=1}^{m k} v_{i}^{*} \iota(a) v_{i}\right\|<2 \epsilon$ for all $a \in \mathcal{F} \cup \Psi_{A}(\mathcal{F})$. Then, for each $a \in \mathcal{F} \cup \Psi_{A}(\mathcal{F})$,

$$
\begin{aligned}
\left\|\phi(a) 1_{B}-\sum_{i} \Psi_{B}\left(v_{i}\right) \iota(a) \Psi_{B}\left(v_{i}^{*}\right)\right\| & =\left\|\phi(a) 1_{B}-\Psi_{B}\left(\sum_{i} v_{i}^{*} \iota\left(\Psi_{A}(a)\right) v_{i}\right)\right\| \\
& =\left\|\phi\left(\Psi_{A}(a)\right) 1_{B}-\Psi_{B}\left(\sum_{i} v_{i}^{*} \iota\left(\Psi_{A}(a)\right) v_{i}\right)\right\| \\
& =\left\|\phi\left(\Psi_{A}(a)\right) 1_{B}-\sum_{i} v_{i}^{*} \iota\left(\Psi_{A}(a)\right) v_{i}\right\| \\
& <2 \epsilon .
\end{aligned}
$$

Therefore

$$
\left\|\phi(a) 1_{B}-\sum_{j} \frac{1}{2}\left[v_{j}^{*} \iota(a) v_{j}+\Psi_{B}\left(v_{j}\right) \iota(a) \Psi_{B}\left(v_{j}^{*}\right)\right]\right\|<2 \epsilon .
$$

Let $v_{j}=w_{j}+i w_{j}^{\prime}$ with $w_{j}, w_{j}^{\prime} \in B$. Then

$$
\begin{aligned}
v_{j}^{*} \iota(a) v_{j}+\Psi_{B}\left(v_{j}\right) \iota(a) \Psi_{B}\left(v_{j}^{*}\right)= & \left(w_{j}^{*}-i w_{j}^{\prime *}\right) \iota(a)\left(w_{j}+i w_{j}^{\prime}\right) \\
& +\left(w_{j}^{*}+i w_{j}^{* *} \iota(a)\left(w_{j}-i w_{j}^{\prime}\right)\right. \\
= & 2 w_{j}^{*} \iota(a) w_{j}+2 w_{j}^{\prime *} \iota(a) w_{j}^{\prime},
\end{aligned}
$$

SO

$$
\left\|\phi(a) 1_{B}-\sum\left(w_{j}^{*} \iota(a) w_{j}+w_{j}^{\prime *} \iota(a) w_{j}^{\prime}\right)\right\|<2 \epsilon .
$$

Letting $V=\left(v_{1}, \ldots, v_{m k}\right)^{t r}, W=\left(w_{1}, \ldots, w_{m k}\right)^{t r}, W^{\prime}=\left(w_{1}^{\prime}, \ldots, w_{m k}^{\prime}\right)^{t r}$, it follows from $V^{*} V=1_{B}$ that $\left(W+i W^{\prime}\right)^{*}\left(W+i W^{\prime}\right)=1_{B}$ and therefore that $W^{*} W+W^{\prime *} W^{\prime}=1_{B} .\left(W, W^{\prime}\right)$ is therefore an isometry in $M_{2 m k \times 1}(B)$ with

$$
\left\|\phi(a) 1_{B}-\left(W, W^{\prime}\right)^{*}\left(1_{2 m k} \otimes \iota(a)\right)\left(W, W^{\prime}\right)\right\|<2 \epsilon
$$

for all $a \in \mathcal{F}$.

Proposition 3. Let $A$ be a unital separable real $C^{*}$-algebra and let $\iota: A \hookrightarrow B$ be a unital full embedding. Then $d_{\iota}$ is nuclearly absorbing.

Proof. The proof of Proposition 2.19 of [6] carries over to the real case so that the result follows from the lemma above. 


\section{RESULTS FROM $K$-THEORY}

3.1. United $K$-theory and the universal coefficient theorem. In this section the relevant $K$-theoretical tools are summarized for later use. The first set of results relates to the united $K$-theory used by Boersema in [3] to prove a universal coefficient theorem for real $C^{*}$-algebras. The basic objects of study in united $K$ theory are $C R T$-modules, first defined by Bousfield in 4 and applied to the $K$ theory of real $C^{*}$-algebras by Boersema in 2 . The $C R T$-modules arising in the $K$-theory of a real $C^{*}$-algebra $A$ are built from the $K$-groups of $A, A \otimes \mathbb{C}$ and $A \otimes T$, where $T=\{f \in C([0,1], \mathbb{C}): f(0)=\overline{f(1)}\}$. It is shown in Proposition 8.0.2 of [9] that if, as in the cases considered in this paper, $K_{1}(A \otimes \mathbb{C})=0$, then the exact $C R T$-module corresponding to $A$ is $C R$-acyclic. It is further shown in Theorem 8.1.2 of 9 that $C R$-acyclic exact $C R T$-modules are equivalent to $C R$ modules, which involve only the real and complex parts of exact $C R T$-modules and are therefore much easier to work with and describe.

A $C R$-module consists of a pair $\left\{M_{*}^{C}, M_{*}^{R}\right\}$ of $\mathbb{Z}$-graded abelian groups with homomorphisms $B: M_{*}^{C} \cong M_{*+2}^{C}, \psi: M_{*}^{C} \cong M_{*}^{C}, B_{R}: M_{*}^{R} \cong M_{*+8}^{R}, \eta: M_{*}^{R} \rightarrow$ $M_{*+1}^{R}, c: M_{*}^{R} \rightarrow M_{*}^{C}$ and $r: M_{*}^{C} \rightarrow M_{*}^{R}$ satisfying various specified relations. If $A$ is a real $C^{*}$-algebra, then such a $C R$-module (with an additional property known as exactness or acyclicity) is given by $M_{*}^{C}=K_{*}(A \otimes \mathbb{C})$ and $M_{*}^{R}=K_{*}(A)$. The map $B$ is the usual Bott periodicity map, the map $B_{R}$ is the real periodicity map, the map $\psi$ is induced by the real-linear automorphism $a+i b \mapsto a-i b$, where $a, b \in A$, the map $c$ is induced by the embedding of $A$ in $A \otimes \mathbb{C}$, the map $r$ is induced by embedding $A \otimes \mathbb{C}$ in $M_{2}(A)$ and the map $\eta$ is obtained by multiplication by the generator of $K_{1}(\mathbb{R})$. Details can be found in [2] and [9].

If $A$ is a real $C^{*}$-algebra such that $A \otimes \mathbb{C}$ is a UHF-algebra with infinitely many $2 \times 2$ matrix factors, the calculations in Theorem 8.4.2 of [1], although stated just for the CAR algebra, apply to this situation and give $K_{i}(A)=0$ for $i=1,2,3,5,6,7$ and $K_{0}(A), K_{4}(A)$ and $K_{0}(A \otimes \mathbb{C})$ all isomorphic to the same dense subgroup $G$ of $\mathbb{R}$. (These results are also easily obtained from the treatment of even abstract cores in section 8.3 of $[9$, which shows that the exact $C R$-module corresponding to $A$ is determined by $K_{0}(A \otimes \mathbb{C})$ and $\psi: K_{0}(A \otimes \mathbb{C}) \rightarrow K_{0}(A \otimes \mathbb{C})$, which are the same as for the real UHF-algebra of the same type as $A$.) In this situation $\psi=1$ on $K_{0}(A \otimes \mathbb{C})$ and $K_{4}(A \otimes \mathbb{C}), \psi=-1$ on $K_{2}(A \otimes \mathbb{C})$ and $K_{6}(A \otimes \mathbb{C})$ and $\eta$ is the zero map. If the isomorphisms $\rho_{A}^{R}: K_{0}(A) \rightarrow G, \rho_{A}^{C}: K_{0}(A \otimes \mathbb{C}) \rightarrow G$ and $\rho_{A}^{H}: K_{0}(A \otimes \mathbb{H}) \rightarrow G$ are chosen with $\rho_{A}^{R}\left(\left[1_{A}\right]\right)=1, \rho_{A}^{C}\left(\left[1_{A \otimes \mathbb{C}}\right]\right)=1$ and $\rho_{A}^{H}\left(\left[1_{A \otimes H}\right]\right)=1$, then $c_{0}$ and $r_{4}$ correspond to the identity map on $G$ and $r_{0}$ and $c_{4}$ to multiplication by 2 (which is an automorphism).

A real version of the Universal Coefficient Theorem of Rosenberg and Schochet [17] was obtained by Boersema in [3]. When restricted to the real parts of the $C R T$-modules, his theorem implies, as described in Corollary 4.9 of [3], that, for real separable $C^{*}$-algebras $A$ and $B$, with $A \otimes \mathbb{C}$ in the bootstrap category $\mathcal{N}$, there is a short exact sequence

$$
0 \rightarrow \operatorname{Ext}_{[,]}\left(K^{C R T}(A), K^{C R T}(B)\right) \stackrel{\kappa_{0}}{\rightarrow} K K(A, B) \stackrel{\gamma_{0}}{\rightarrow}\left[K^{C R T}(A), K^{C R T}(B)\right] \rightarrow 0,
$$

where $[M, N]$ denotes the graded group of $C R T$-morphisms from the $C R T$-module $M$ to the $C R T$-module $N, \operatorname{Ext}_{[,]}(M, N)$ denotes the associated derived functor and $\kappa_{0}$ has degree -1 . The homomorphism $\gamma_{0}$ is given by $\gamma_{0}(x)(y)=\alpha(y, x)$, where $\alpha$ is the intersection product from $K_{0}(A \otimes D) \times K K(A, B) \cong K K(\mathbb{R}, A \otimes D) \times K K(A, B)$ 
to $K K(\mathbb{R}, B \otimes D) \cong K_{0}(B \otimes D)$. An extension to the case where $B$ is $\sigma$-unital, but not separable, is given in the appendix to this paper.

As noted above, for algebras such as those with $K_{1}(A \otimes \mathbb{C})=K_{1}(B \otimes \mathbb{C})=0$, which are necessarily $C R$-acyclic, the category of $C R T$-modules can be replaced by that of $C R$-modules. In the case where the only non-zero $K$-groups of $A, A \otimes \mathbb{C}$, $B$ and $B \otimes \mathbb{C}$ are in even degrees, it follows from the degree -1 property of $\kappa_{0}$ (implying that the exact sequence involves components $\operatorname{Ext}\left(K_{*}(A), K_{*+1}(B)\right)$ and $\operatorname{Ext}\left(K_{*}(A \otimes \mathbb{C}), K_{*+1}(B \otimes \mathbb{C})\right)$ that $\operatorname{Ext}_{[,]}\left(K^{C R T}(A), K^{C R T}(B)\right)=0$. Therefore in this case $\gamma_{0}$ is an isomorphism from $K K(A, B)$ onto the set of $C R$-morphisms from $K^{C R}(A)$ to $K^{C R}(B)$. When $A \otimes \mathbb{C}$ and $B \otimes \mathbb{C}$ are UHF-algebras with infinitely many $2 \times 2$ matrix factors, these morphisms are determined by the values on $\left[1_{A}\right] \in K_{0}(A)$, so there exists $\beta \in K K(A, B)$ with $\beta_{*}\left(\left[1_{A}\right]\right)=\alpha\left(\left[1_{A}\right], \beta\right)=\left[1_{B}\right]$.

3.2. $K^{C R}$-triples. In this section we establish notation for the appropriate counterparts to the $\underline{K}$-triples introduced in Section 3.3 of $\left[\underline{6}\right.$. The real $C^{*}$-algebras $A$ considered in the present paper have, up to periodicity, no non-zero $K$-groups other than $K_{0}(A), K_{4}(A)$ and $K_{0}(A \otimes \mathbb{C})$. Therefore the $C R$-module elements with which we are concerned can all be realised as differences of classes of projections from

$$
\operatorname{Proj}^{C R}(A)=\operatorname{Proj}\left(A \otimes \mathcal{K}_{\mathbb{R}}\right) \cup \operatorname{Proj}\left(A \otimes \mathbb{C} \otimes \mathcal{K}_{\mathbb{R}}\right) \cup \operatorname{Proj}\left(A \otimes \mathbb{H} \otimes \mathcal{K}_{\mathbb{R}}\right) .
$$

This definition suffices in the limited circumstances considered here, but it will need modification to cover more general situations.

As in Definition 3.7 of [6], a $K^{C R}$-triple $(\mathcal{P}, \mathcal{G}, \delta)$ for $A$ (of the form considered here) consists of finite subsets $\mathcal{P} \subset \operatorname{Proj}^{C R}(A)$ and $\mathcal{G} \subset A$ and a $\delta>0$ chosen such that whenever $\phi$ is a completely positive contraction which is $\delta$-multiplicative on $\mathcal{G}$, then $\frac{1}{2}$ does not belong to the spectrum of $(\phi \otimes \operatorname{id} \mathcal{K})(p)$ for each $p \in \mathcal{P}$ (where $\phi$ is also used for the extensions $\phi \otimes$ id to $A \otimes \mathbb{C}$ and $A \otimes \mathbb{H}$ ). As in Lemma 3.8 of [6], every finite set $\mathcal{P}$ of projections in $\operatorname{Proj}^{C R}(A)$ can be augmented to a $K^{C R_{-}}$ triple for $A$. Then, as in Definition 3.9 of [6] for any $K^{C R}$-triple $(\mathcal{P}, \mathcal{G}, \delta)$ for $A$ and any completely positive contraction which is $\delta$-multiplicative on $\mathcal{G}, \phi_{\sharp}: \mathcal{P} \rightarrow$ $K_{0}(B) \cup K_{0}(B \otimes \mathbb{C}) \cup K_{0}(B \otimes \mathbb{H})$ can be defined by

$$
\phi_{\sharp}(p)=\left[\chi_{0}\left(\left(\phi \otimes \operatorname{id}_{\mathcal{K}}\right)(p)\right)\right],
$$

where $\chi_{0}$ is the characteristic function of $\left(\frac{1}{2}, 1\right]$.

As in Lemma 3.10 of [6], if $A, B$ and $C$ are unital real $C^{*}$-algebras, $(\mathcal{P}, \mathcal{G}, \delta)$ is a $K^{C R}$-triple for $A, \phi: A \rightarrow B$ is a completely positive contraction which is $\delta$-multiplicative on $\mathcal{G}$ and $j: B \rightarrow C$ is a unital ${ }^{*}$-homomorphism, then

$$
(j \phi)_{\sharp}(p)=j_{*} \phi_{\sharp}(p)
$$

for all $p \in \mathcal{P}$.

3.3. The $K$-theory of products. The techniques of Lin and of Dadarlat and Eilers to be used in Section 4 involve making approximate homomorphisms exact by passing to a sequence algebra $\prod B_{i} / \sum B_{i}$, where $\prod B_{i}$ is the $\ell_{\infty}$ product of the $C^{*}$-algebras $B_{i}$ and $\sum B_{i}$ is their $c_{0}$ sum. It follows from Theorem 4.10 and related results from [6] that $K_{1}(C)=0$ when $C=\prod B_{i} / \sum B_{i}$ where either $B_{i}$ are UHF-algebras or are of the form $\prod D_{i} / \sum D_{i}$, where $D_{i}$ are UHF-algebras. This will enable the results described in [6] to be transferred to the situation of interest in this paper. 
Let $B$ be a real unital $C^{*}$-algebra satisfying $K_{1}(B \otimes \mathbb{C})=0, K_{0}(B \otimes \mathbb{C})=$ $2 K_{0}(B \otimes \mathbb{C})$ is torsion-free,

$$
K_{0}\left(\ell_{\infty}(B \otimes \mathbb{C})\right) \cong\left\{\left(x_{i}\right) \in \prod K_{0}(B \otimes \mathbb{C}) \mid(\exists M \in \mathbb{N})(\forall i \in \mathbb{N})-M[1] \leq x_{i} \leq M[1]\right\}
$$

and

$$
0 \rightarrow K_{0}\left(c_{0}(B \otimes \mathbb{C})\right) \rightarrow K_{0}\left(\ell_{\infty}(B \otimes \mathbb{C})\right) \rightarrow K_{0}\left(\ell_{\infty}(B \otimes \mathbb{C}) / c_{0}(B \otimes \mathbb{C})\right) \rightarrow 0
$$

is exact. Assume further that, if $\psi_{i}$ is the involution of $K_{i}(B \otimes \mathbb{C})$ arising from the map $a+i b \mapsto a-i b$ for $a, b \in B$, then $\psi_{i}=1$ for $i=0,4$ and $\psi_{i}=-1$ for $i=2,6$. It follows that if $\Psi_{i}$ is the corresponding involution on $K_{i}\left(\ell_{\infty}(B \otimes \mathbb{C})\right)$ or $K_{i}\left(\ell_{\infty}(B \otimes \mathbb{C}) / c_{0}(B \otimes \mathbb{C})\right)$, then $\Psi_{i}=1$ for $i=0,4$ and $\Psi_{i}=-1$ for $i=2,6$. The $C R T$-modules $K^{C R T}\left(\ell_{\infty}(B)\right)$ and $K^{C R T}\left(\ell_{\infty}(B) / c_{0}(B)\right)$ are therefore $h_{*}$-acyclic in the terminology of Chapter 6 of $[9$. Noting that, by Proposition 8.0.2 of [9], such modules are $C R$-acyclic and therefore, by Theorem 8.1.1 of [9], are isomorphic under the forgetful functor to $h_{*}$-acyclic exact $C R$-modules, Theorem 8.4.2 of $[9$ yields that $K_{i}\left(\ell_{\infty}(B)\right)=K_{i}\left(\ell_{\infty}(B) / c_{0}(B)\right)=0$ for $i=1,2,3,5,6,7$ and $K_{i}\left(\ell_{\infty}(B)\right) \cong$ $K_{i}\left(\ell_{\infty}(B \otimes \mathbb{C})\right)$ and $K_{i}\left(\ell_{\infty}(B) / c_{0}(B)\right) \cong K_{i}\left(\ell_{\infty}(B \otimes \mathbb{C}) / c_{0}(B \otimes \mathbb{C})\right)$ for $i=0,4$. This argument applies when $B$ is either of the form $f A f$ for a projection $f$ in $A$ or $f \ell_{\infty}(A) / c_{0}(A) f$ for a projection $f$ in $\ell_{\infty}(A) / c_{0}(A)$, where $A \otimes \mathbb{C}$ is a UHF-algebra with infinitely many $2 \times 2$ matrix factors. Furthermore, when the argument applies to $B$, it applies to $\ell_{\infty}(B)$ and to $\ell_{\infty}(B) / c_{0}(B)$.

\section{UNIQUENESS}

The aim of this section is to produce a version of Theorem 6.7 of [6] which is relevant in the present context. There are added complications in extending results from the complex to the real case, and, for this reason, the results here are not given in the same generality as the results in [6]. A general classification theorem for real nuclear $C^{*}$-algebras would have to address the more general situation.

The results and proofs closely follow those in [6] and 14]. In particular, the following result is based on Theorem 4.5 of [6] and Theorem 5.6.4 of [14].

Proposition 4. Let $A$ be a unital separable, nuclear real $C^{*}$-algebra, let $B$ be a unital real $C^{*}$-algebra and let $\iota: A \hookrightarrow B$ be a unital full embedding. Let $\gamma: A \rightarrow$ $M\left(\mathcal{K}_{\mathbb{R}}(H) \otimes B\right)$ be defined by $\gamma(a)=\operatorname{diag}(\iota(a), \iota(a), \ldots)=1 \otimes \iota(a)$ and, for $n \in \mathbb{N}$, let $\gamma_{n}(a)=\left(e_{n} \otimes 1\right) \gamma(a)$, where $e_{n}=\operatorname{diag}(1,1, \ldots, 1,0,0, \ldots) \in \mathcal{K}_{\mathbb{R}}(H)$.

Suppose that $\alpha, \beta: A \rightarrow B$ are ${ }^{*}$-homomorphisms with $[\alpha]=[\beta]$ in $K K(A, B)$. Then, for any finite subset $\mathcal{F} \subset A$ and any $\epsilon>0$ there exists $n \in \mathbb{N}$ and a unitary $u \in M_{n+1}(B)$ such that

$$
\left\|\operatorname{udiag}\left(\alpha(a), \gamma_{n}(a)\right) u^{*}-\operatorname{diag}\left(\beta(a), \gamma_{n}(a)\right)\right\|<\epsilon
$$

for all $a \in \mathcal{F}$. Moreover, it can be arranged that

$$
u \operatorname{diag}\left(\alpha(1), \gamma_{n}(1)\right) u^{*}=\operatorname{diag}\left(\beta(1), \gamma_{n}(1)\right) .
$$

Proof. By Proposition $3, d_{\iota}: A \rightarrow M\left(\mathcal{K}_{\mathbb{R}}(H) \otimes B\right)$ is absorbing. The proofs of Lemma 5.6.2, Lemma 5.6.3 and Theorem 5.6.4 of [14] (with $p(t)=V(t) e_{N} V(t)^{*}$ in the latter) therefore carry across to the real setting, provided that instead of first simplifying to the case $\mathcal{F} \subset A_{s a}$, elements of the simplified $\mathcal{F}$ are also allowed to be skew-adjoint. The non-unital case is handled, as in Corollary 4.4 and Theorem 4.5 of [6], by including an extra zero summand until the final step. The final adjustment 
to give $u \operatorname{diag}\left(\alpha(1), \gamma_{n}(1)\right) u^{*}=\operatorname{diag}\left(\beta(1), \gamma_{n}(1)\right)$ uses a small perturbation of $u$ (cut down in the non-unital case), as in the proof of Theorem 4.5 of [6].

Proposition 5. Let $A$ be a real $C^{*}$-algebra for which the complexification $A \otimes \mathbb{C}$ is isomorphic to a UHF-algebra with infinitely many $2 \times 2$ matrix factors and let $B$ be isomorphic to $\ell_{\infty}(C) / c_{0}(C)$, where $C$ is isomorphic to either $f$ Af for some projection $f \in A$ or to $f \ell_{\infty}(A) / c_{0}(A) f$ for some projection $f \in \ell_{\infty}(A) / c_{0}(A)$. Then, for any finite subset $\mathcal{F} \subset A$ and any $\epsilon>0$, there exists $n \in \mathbb{N}$ with the following property. If $\iota: A \rightarrow B$ is a unital embedding and if $\phi, \psi: A \rightarrow B$ are ${ }_{\text {* }}$ homomorphisms such that $\phi_{*}=\psi_{*}$ as $C R$-module homomorphisms from $K^{C R}(A)$ to $K^{C R}(B)$ and $\phi(1)$ is unitarily equivalent to $\psi(1)$, there exists a unitary $u \in$ $M_{n+1}(B)$ such that

$$
\left\|u\left(\begin{array}{cc}
\phi(a) & \\
& n \iota(a)
\end{array}\right) u^{*}-\left(\begin{array}{cc}
\psi(a) & \\
& n \iota(a)
\end{array}\right)\right\|<\epsilon
$$

for all $a \in \mathcal{F}$. It can be arranged that $u(\phi(1) \oplus n 1) u^{*}=\psi(1) \oplus n 1$.

Proof. Following the proof of Theorem 4.12 of [6], for each $m$ let $\iota_{m}, \phi_{m}, \psi_{m}$ satisfy the conditions of the theorem but with

$$
\max _{a \in \mathcal{F}}\left\|u\left(\begin{array}{cc}
\phi_{m}(a) & \\
& m \iota_{m}(a)
\end{array}\right) u^{*}-\left(\begin{array}{ll}
\psi_{m}(a) & \\
& m \iota_{m}(a)
\end{array}\right)\right\| \geq \epsilon
$$

for every unitary $u \in M_{m+1}(B)$. Continuing as in [6], define $\dot{\Phi}, \dot{\Psi}, \dot{I}: A \rightarrow$ $\ell_{\infty}(B) / c_{0}(B)$ and use the results on products from Section 3.3 to deduce that $\dot{\Phi}_{*}=\dot{\Psi}_{*}$. Then note that the Universal Coefficient Theorem for united $K$-theory, from [3] and the appendix to this paper, can be applied to give $[\dot{\Phi}]=[\dot{\Psi}]$ in $K K\left(A, \ell_{\infty}(B) / c_{0}(B)\right)$. As in [6], this enables Proposition 4 to be applied to give a contradiction.

Proposition 6. Let $A$ be a real $C^{*}$-algebra for which the complexification $A \otimes \mathbb{C}$ is isomorphic to a UHF-algebra with infinitely many $2 \times 2$ matrix factors and let $B$ be isomorphic to either $A$ or $\ell_{\infty}(A) / c_{0}(A)$. Then, for any finite subset $\mathcal{F} \subset A$ and any $\epsilon>0$, there exist $n \in \mathbb{N}$ and a $K^{C R}$-triple $(\mathcal{P}, \mathcal{G}, \delta)$ with the following property. If $f \in B$ is a projection and $\phi, \psi, \tau: A \rightarrow f B f$ are completely positive contractions which are $\delta$ multiplicative on $\mathcal{G}$, with $\tau$ unital, with $\phi(1)$ and $\psi(1)$ unitarily equivalent projections and with $\phi_{\sharp}(p)=\psi_{\sharp}(p)$ in $K^{C R}(f B f)$ for all $p \in \mathcal{P}$, there exists a unitary $u \in M_{n+1}(f B f)$ such that

$$
\left\|u\left(\begin{array}{cc}
\phi(a) & \\
& n \tau(a)
\end{array}\right) u^{*}-\left(\begin{array}{cc}
\psi(a) & \\
& n \tau(a)
\end{array}\right)\right\|<\epsilon
$$

for all $a \in \mathcal{F}$. It can be arranged that $u(\phi(1) \oplus n f) u^{*}=\psi(1) \oplus n f$.

Proof. The proof exactly follows that of Theorem 4.15 of [6], using Proposition 5 instead of Theorem 4.12 of [6] and using the results on products from Section 3.3 . Instead of the algebra $C$ used in [6], the algebras $\mathcal{K}_{\mathbb{R}}, \mathbb{C} \otimes \mathcal{K}_{\mathbb{R}}$ and $\mathbb{H} \otimes \mathcal{K}_{\mathbb{R}}$ are used to show that the maps $\dot{\Phi}_{*}, \dot{\Psi}_{*}: K^{C R}(A) \rightarrow K^{C R}\left(\ell_{\infty}(f B f) / c_{0}(f B f)\right)$ are equal.

The following real analogue of Lemma 6.10 of [12] plays a fundamental role in the proof of Proposition 8 . The presentation here follows that in Lemma 6.5 of [6]. 
Proposition 7. Let $A$ be be a real $C^{*}$-algebra for which the complexification $A \otimes \mathbb{C}$ is isomorphic to a UHF-algebra. For any $n \geq 1$, any finite subset $\mathcal{F} \subset A$ and any $\epsilon>0$, there are projections $p, q \in A$ with $(1-p) A(1-p) \cong M_{n}(q A q)$ and $[p] \leq[q]$, a unital finite dimensional $C^{*}$-subalgebra $C$ of $q A q$, a unital completely positive contractive map $\eta: A \rightarrow C$ and a unital ${ }^{*}$-homomorphism $\mu: p A p \oplus M_{n}(C) \rightarrow A$ restricting to the natural inclusion of $p A p$ in $A$, such that $\nu: A \rightarrow p A p \oplus M_{n}(C)$ defined by $\nu(a)=\operatorname{pap} \oplus\left(\eta(a) \otimes 1_{n}\right)$ is $\epsilon$-multiplicative on $\mathcal{F}$ and $\|\mu \nu(a)-a\|<\epsilon$ for all $a \in \mathcal{F}$.

Proof. By [23] $A$ has tracial rank zero. As in [6] there exist a projection $p \in A$ and a finite dimensional real $C^{*}$-algebra $C$ with $1-p \in C \subset(1-p) A(1-p)$ such that, for all $a \in \mathcal{F},\|[p, a]\|<\epsilon,(1-p) a(1-p) \in_{\epsilon} C$ and $(n+2)[p] \leq[1]$. If $e_{1}, \ldots, e_{k}$ are the minimal central projections of $C$, then, for each $i, e_{i} C e_{i}$ is isomorphic to $M_{m(i)}\left(F_{i}\right)$ where $F_{i}$ is isomorphic to $\mathbb{R}, \mathbb{C}$ or $\mathbb{H}$. When $F_{i}=\mathbb{R}$ or $F_{i}=\mathbb{H}, e_{i} A e_{i}$ factorises as $M_{m(i)}\left(F_{i}\right) \otimes B_{i}$, where $B_{i}$ is the relative commutant of $e_{i} C e_{i}$ in $e_{i} A e_{i}$. When $F_{i}=\mathbb{C}, e_{i}$ is no longer a minimal central projection in the complexification but can be written as $e_{i 1}+e_{i 2}$, where $e_{i 1}$ and $e_{i 2}$ are minimal and central, with $\Phi_{A}\left(e_{i 1}\right)=e_{i}-e_{i 1}=e_{i 2}$, where $\Phi_{A}$ is the involutory antiautomorphism of $A \otimes \mathbb{C}$ corresponding to $A$. If the relative commutant of $e_{i 1}(C \otimes \mathbb{C}) e_{i 1}$ in $e_{i 1}(A \otimes \mathbb{C}) e_{i 1}$ is $B_{i 1}$, then $B_{i}=\left\{b+\Phi_{A}\left(b^{*}\right): b \in B_{i 1}\right\}$ is isomorphic to $B_{i 1}$ and is contained in the relative commutant of $e_{i} C e_{i}$ in $e_{i} A e_{i}$. Thus, in all cases, $B_{i}$ is tracially AF, being isomorphic to a corner of $A$ or $A \otimes \mathbb{C}$, and is contained in the relative commutant of $e_{i} C e_{i}$ in $e_{i} A e_{i}$. This algebra (with $K_{0}\left(B_{i}\right)$ isomorphic to a dense subgroup of $\mathbb{R}$ ) satisfies the conclusions of Lemma 6.4 of [6]. The remaining part of the proof of Lemma 6.5 of [6] then carries through without change, using $E=\frac{1}{2}\left(E^{C}+\Phi_{A} \circ * \circ E^{C}\right)$ for the completely positive contraction from $A$ to $C$, where $E^{C}$ is the corresponding contraction from $A \otimes \mathbb{C}$ onto $C \otimes \mathbb{C}$.

Proposition 8. Let $A$ be a real $C^{*}$-algebra for which the complexification $A \otimes \mathbb{C}$ is isomorphic to a UHF-algebra with infinitely many $2 \times 2$ matrix factors. Then, for any finite subset $\mathcal{F} \subset A$ and any $\epsilon>0$, there exists a $K^{C R}$-triple $(\mathcal{P}, \mathcal{G}, \delta)$ with the following property. For any two unital completely positive contractions $\phi, \psi: A \rightarrow A$ which are $\delta$-multiplicative on $\mathcal{G}$ and with $\phi_{\sharp}(p)=\psi_{\sharp}(p)$ for all $p \in \mathcal{P}$, there exists a unitary $u \in A$ such that

$$
\left\|u \phi(a) u^{*}-\psi(a)\right\|<\epsilon
$$

for all $a \in \mathcal{F}$.

Proof. The proof exactly follows that of Theorem 6.7 of [ 6 , using Propositions 7 and 6 instead of Lemma 6.5 and Theorem 4.15 of [6]. The proof requires $A$ and $\ell_{\infty}(A) / c_{0}(A)$ to have a cancellation of projections. In the case of $A$ this property follows from Proposition 5.1 and Lemma 6.3 of [22]. It then follows for $\ell_{\infty}(A) / c_{0}(A)$ by using standard approximation techniques to lift projections $E, F$ and a partial isometry $V$ with $E=V V^{*}$ and $F=V^{*} V$ in the quotient algebra to sequences $\left(e_{n}\right),\left(f_{n}\right),\left(v_{n}\right)$ which are ultimately projections or partial isometries and then by using the fact that close projections are unitarily equivalent to establish that eventually $1-e_{n}$ is equivalent to $1-f_{n}$ and thus $1-E$ is equivalent to $1-F$. 


\section{EXISTENCE}

The aim of this section is to produce a version of Lemma 6.4 .7 of [14]. The approach is closely modeled on Section 5 of [6]. This uses the description of $K K(A, B)$ as homotopy equivalence classes of Cuntz pairs of representations $(\phi, \psi): A \rightarrow$ $M\left(\mathcal{K}_{\mathbb{R}}(H) \otimes B\right)$ with $\phi(a)-\psi(a) \in \mathcal{K}_{\mathbb{R}}(H) \otimes B$ for all $a \in A$. The first lemma is the real version (for nuclear $A$ ) of Lemma 3.1 of [6].

Lemma 9. Let $A$ be a unital, separable, nuclear, real $C^{*}$-algebra, let $B$ be a unital, real $C^{*}$-algebra, let $\theta^{\prime}$ be a faithful representation of $A$ on a real Hilbert space $H$, let $H^{\prime}$ be an infinite dimensional real Hilbert space and let $\theta: A \rightarrow M\left(\mathcal{K}_{\mathbb{R}}\left(H \otimes H^{\prime}\right) \otimes B\right)$ factor as

$$
A \stackrel{\theta^{\prime} \otimes 1}{\rightarrow} \mathcal{L}_{\mathbb{R}}\left(H \otimes H^{\prime}\right) \stackrel{-\otimes 1}{\rightarrow} \mathcal{L}_{\mathbb{R}}\left(H \otimes H^{\prime}\right) \otimes M(B) \rightarrow M\left(\mathcal{K}_{\mathbb{R}}\left(H \otimes H^{\prime}\right) \otimes B\right) .
$$

Then any $\alpha \in K K(A, B)$ is represented by a Cuntz pair of the form $(\rho, \hat{\theta})$, where $\hat{\theta}=0 \oplus \theta: A \rightarrow M\left(\mathcal{K}_{\mathbb{R}}\left(\left(H \otimes H^{\prime}\right) \oplus\left(H \otimes H^{\prime}\right)\right) \otimes B\right)$.

Proof. Let $A, B$ and $H$ be given trivial $\mathbb{Z}_{2}$-gradings. If $(\mathcal{E}, F)$ is a real Kasparov bimodule representing $\alpha$ (for which $\mathcal{E}$ will be nuclear), then by adding degenerate modules, as detailed in Proposition 2.6 of [19], there exists $F_{1}$ (of degree 1) such that $\left(\left(\mathcal{E} \hat{\otimes} H^{\prime}\right) \oplus\left(H \hat{\otimes} H^{\prime} \hat{\otimes} B\right), F_{1}\right)$ also represents $\alpha$, where $H^{\prime}=H_{+}^{\prime} \oplus H_{-}^{\prime}$ is a $\mathbb{Z}_{2^{-}}$ graded real Hilbert space with $H_{+}^{\prime}$ and $H_{-}^{\prime}$ infinite dimensional, where the tensor products are $\mathbb{Z}_{2}$-graded and where the $A$-action on $H \hat{\otimes} H^{\prime} \hat{\otimes} B$ is given by $\theta^{\prime} \hat{\otimes} 1 \hat{\otimes} 1$. Then, noting that the arguments given by Kasparov in [11] apply to the real case with a $\mathbb{Z}_{2}$-action, the arguments in Theorem 1.5 and Remark 1.10 of 19 show that there is a unitary $u$ of degree zero in $\mathcal{L}_{B}\left(\left(\mathcal{E} \hat{\otimes} H^{\prime}\right) \oplus\left(H \hat{\otimes} H^{\prime} \hat{\otimes} B\right), H \hat{\otimes} H^{\prime} \hat{\otimes} B\right)$ intertwining the $A$-actions modulo the compacts. Thus $(\mathcal{E}, F)$ represents the same $K K$ element as $\left(H \hat{\otimes} H^{\prime} \hat{\otimes} B, u F_{1} u^{*}\right)$.

Following Theorem 4.1.8 of [10], assume that $u F_{1} u^{*}=\left(u F_{1} u^{*}\right)^{*},-1 \leq u F_{1} u^{*} \leq$ 1 and $u F_{1} u^{*}=\left(\begin{array}{cc}0 & x \\ x^{*} & 0\end{array}\right)$ relative to the grading decomposition of $H \hat{\otimes} H^{\prime} \hat{\otimes} B$. The map $\bar{\theta}: a \mapsto \theta^{\prime}(a) \otimes 1 \otimes 1$ is of degree 0 , and hence there exist $\theta_{+}: A \rightarrow H \otimes H_{+}^{\prime} \otimes B$, $\theta_{-}: A \rightarrow H \otimes H_{-}^{\prime} \otimes B$, both defined by $a \mapsto \theta^{\prime}(a) \otimes 1 \otimes 1$ (and hence identifiable with $\theta$ from the statement of the lemma when $H_{+}^{\prime}$ and $H_{-}^{\prime}$ are identified with $\left.H^{\prime}\right)$ with $\bar{\theta}=\left(\begin{array}{cc}\theta_{+} & 0 \\ 0 & \theta_{-}\end{array}\right)$. Choose isometries $W_{1}, W_{2} \in \mathcal{L}_{B}\left(\left(H \otimes H^{\prime}\right) \otimes B\right)$ with $W_{1} W_{1}^{*}+W_{2} W_{2}^{*}=1$ and $W_{1}^{*} W_{2}=0$ and let $T:\left(\left(H \otimes H^{\prime}\right) \otimes B\right) \oplus\left(\left(H \otimes H^{\prime}\right) \otimes\right.$ $B) \rightarrow\left(H \otimes H^{\prime}\right) \otimes B$ be the isomorphism of Hilbert $B$-modules given by $T(x, y)=$ $W_{1} x+W_{2} y$ for $x, y \in\left(H \otimes H^{\prime}\right) \otimes B$. Then, as in the proof of Theorem 4.1.8 of [10], $\alpha$ is represented by $\left(H \hat{\otimes} H^{\prime} \hat{\otimes} B,\left(\begin{array}{ll}0 & 1 \\ 1 & 0\end{array}\right)\right)$ with $A$-action $\left(\begin{array}{cc}A d u^{*} \circ \psi_{+} & 0 \\ 0 & \psi_{-}\end{array}\right)$, where $\psi_{+}=T\left(\theta_{+} \oplus 0\right) T^{*}$ and $\psi_{-}=T\left(\theta_{-} \oplus 0\right) T^{*}$. This corresponds, as in Lemma 4.1.7 of [10, to the Cuntz pair $\left(A d u^{*} \circ \psi_{+}, \psi_{-}\right)$, which is isomorphic to $\left(A d\left(T^{*} u^{*} T\right) \circ \hat{\theta}_{+}, \hat{\theta}_{-}\right)$ and thus to $\left(\operatorname{Ad}\left(T^{*} u^{*} T\right) \circ \hat{\theta}_{+}, \hat{\theta}\right)$.

The next lemma prepares for obtaining an analogue of Theorem 5.4 of $[6]$.

Lemma 10. Let $A$ be a real $C^{*}$-algebra such that $A \otimes \mathbb{C}$ is isomorphic to a UHFalgebra and let $B$ be a unital real $C^{*}$-algebra. Then there exists an admissible scalar 
representation

$$
\theta: A \stackrel{\theta^{\prime} \otimes 1}{\rightarrow} \mathcal{L}_{\mathbb{R}}\left(H \otimes H^{\prime}\right) \stackrel{-\otimes 1}{\rightarrow} \mathcal{L}_{\mathbb{R}}\left(H \otimes H^{\prime}\right) \otimes M(B) \rightarrow M\left(\mathcal{K}_{\mathbb{R}}\left(H \otimes H^{\prime}\right) \otimes B\right),
$$

a sequence $r_{n}$ of integers and a sequence $e_{n} \in M_{r_{n}}(B)$ of projections such that $e_{n}$ is an approximate unit for $\mathcal{K}_{\mathbb{R}}\left(H \otimes H^{\prime}\right) \otimes B$ and $\left[e_{n}, \theta(a)\right] \rightarrow 0$ for any $a \in A$.

Proof. Let $\theta^{\prime}$ be the restriction to $A$ of the GNS representation of $A \otimes \mathbb{C}$ associated with the unique tracial state on $A \otimes \mathbb{C}$, regarding the GNS representation space $H$ as a real Hilbert space. If $A \otimes \mathbb{C}=\lim (A \otimes \mathbb{C})_{n}$ where each $(A \otimes \mathbb{C})_{n}$ is a matrix algebra, then let $P_{n}$ be the real orthogonal projection onto the subspace of $H$ associated with $(A \otimes \mathbb{C})_{n}$, let $Q_{n}$ be the orthogonal projection onto the subspace spanned by the first $n$ elements of an orthonormal basis for $H^{\prime}$ and let $e_{n}=P_{n} \otimes Q_{n} \otimes 1 \in$ $M\left(\mathcal{K}_{\mathbb{R}}\left(H \otimes H^{\prime}\right) \otimes B\right)$. It can then be checked that the result holds when $r_{n}$ is defined by $\left(P_{n} \otimes Q_{n}\right) \mathcal{K}_{\mathbb{R}}\left(H \otimes H^{\prime}\right)\left(P_{n} \otimes Q_{n}\right)=M_{r_{n}}(\mathbb{R})$.

Proposition 11. Let $A$ be a real $C^{*}$-algebra with complexification $A \otimes \mathbb{C}$ isomorphic to a UHF-algebra with infinitely many $2 \times 2$ matrix factors, let $B$ be a unital real $C^{*}$-algebra and let $\alpha \in K K(A, B)$. For any $K^{C R}$-triple $(\mathcal{P}, \mathcal{G}, \delta)$ there exist $N$ and completely positive contractions

$$
\gamma: A \rightarrow M_{N}(B) \text { and } \mu: A \rightarrow M_{N}\left(\mathbb{R} 1_{B}\right)
$$

which are $\delta$-multiplicative on $\mathcal{G}$ and satisfy

$$
\gamma_{\sharp}(p)-\mu_{\sharp}(p)=\alpha_{*}[p]
$$

for all $p \in \mathcal{P}$. It can be arranged that $\sigma(1)$ and $\mu(1)$ are both projections.

Proof. Let $\theta, r_{n}$ and $e_{n}$ be given by Lemma 10 and, using Lemma 9], let $(\rho, \hat{\theta})$ be a Cuntz pair representing $\alpha$. Following the proof of Theorem 5.4 of [6], let $f_{n}=e_{n} \oplus e_{n} \in M_{2 r_{n}}(B)$ and let $\sigma_{n}=f_{n} \rho f_{n}$ and $\theta_{n}=f_{n} \hat{\theta} f_{n}$. Noting that Lemmas $3.13,5.1,5.2$ and 5.3 of 6 remain true in the real case, the remainder of the proof of Theorem 5.4 of [6] then shows that, for sufficiently large $N, \sigma_{N}$ and $\theta_{N}$ are $\delta$-multiplicative on $\mathcal{G}$, with $\theta_{N}(a) \in M_{2 r_{N}}\left(\mathbb{R} 1_{B}\right)$, and $\left(\sigma_{N}\right)_{\sharp}(p)-\left(\theta_{N}\right)_{\sharp}(p)=\alpha_{*}[p]$ for all $p \in \mathcal{P}$. The result therefore holds with $\gamma=\sigma_{N}$ and $\mu=\theta_{N}$.

The following result closely follows Lemma 6.4.7 of [14].

Proposition 12. Let $A$ be a real $C^{*}$-algebra with complexification $A \otimes \mathbb{C}$ isomorphic to a UHF-algebra with infinitely many $2 \times 2$ matrix factors and let $R$ be the real UHFalgebra of the same type as $A \otimes \mathbb{C}$. Let $\rho_{R}: K_{0}(R) \cup K_{0}(R \otimes \mathbb{C}) \cup K_{0}(R \otimes \mathbb{H}) \rightarrow G \subset \mathbb{R}$ restrict to the isomorphism $\rho_{R}^{R}$ from $K_{0}(R)$ to $G \subset \mathbb{R}$ mapping $\left[1_{R}\right]$ to 1 , to the isomorphism $\rho_{R}^{C}$ from $K_{0}(R \otimes \mathbb{C})$ to $G$ mapping $\left[1_{R \otimes \mathbb{C}}\right]$ to 1 , and to the isomorphism $\rho_{R}^{H}$ from $K_{0}(R \otimes \mathbb{H})$ to $G$ mapping $\left[1_{R \otimes \mathbb{H}]}\right.$ to 1 . Let $\rho_{A}$ denote the corresponding map from $K_{0}(A) \cup K_{0}(A \otimes \mathbb{C}) \cup K_{0}(A \otimes \mathbb{H})$ to $G$. Then, for any finite subset $\mathcal{P} \subset$ $\operatorname{Proj}^{C R}(A)$, finite $\mathcal{G} \subset A$ and $\delta>0$, there exists a completely positive contraction $\Phi: A \rightarrow R$, which is $\delta$-multiplicative on $\mathcal{G}$, such that $\rho_{R}\left(\Phi_{\sharp}(p)\right)=\rho_{A}([p])$ for each $p \in \mathcal{P}$.

Proof. Assume that $1_{A} \in \mathcal{P}$ and, by augmenting $\mathcal{G}$ and reducing $\delta$ if necessary, that $(\mathcal{P}, \mathcal{G}, \delta)$ is a $K^{C R}$-triple. By further reducing $\delta$ and augmenting $\mathcal{G}$ if necessary it can be arranged, as in the proof of Lemma 6.11 of [6], that if $p \in \mathcal{P}$ and $[p]=\frac{a}{b}\left[1_{A}\right]$ with $a, b \in \mathbb{N}$, then $\rho_{\sharp}(p)=\frac{a}{b} \rho_{\sharp}\left(1_{A}\right)$ for any completely positive contraction $\rho$ which is $\delta$-multiplicative on $\mathcal{G}$. 
Let $\beta \in K K(A, R)_{+}$satisfy $\beta_{*}\left(\left[1_{A}\right]\right)=\left[1_{R}\right]$, as described at the end of Section 3.1, for which $\rho_{R}^{R} \circ \beta_{*}=\rho_{A}^{R}$. Then, using Proposition [11, let $\gamma, \mu$ be contractions, which are $\frac{1}{2} \delta$-multiplicative on $\mathcal{G}$, with $\gamma_{\sharp}(p)-\mu_{\sharp}(p)=\beta_{*}[p]$ for all $p \in \mathcal{P}$ and with $q=\gamma\left(1_{A}\right)$ and $q^{\prime}=\mu\left(1_{A}\right)$ both projections in $M_{N}(R)$. If $s=\rho_{R}([q])$, then $s=1+t$, where $t=\rho_{R}\left(\left[q^{\prime}\right]\right)$ is non-negative. Let $t=\frac{a}{b}$, where $a, b$ are coprime integers, and let $M$ be a power of 2 greater than or equal to both $a+b$ and $t(a+b)$. Let $k$ be the minimal natural number with $k \geq \frac{M}{a+b}$ (so that $\frac{M}{a}=\frac{M}{t(a+b)}+\frac{M}{a+b} \geq 1+\frac{M}{a+b}>k$ and $\left.\frac{2 M}{a+b} \geq 1+\frac{M}{a+b}>k\right)$. Further, note that

$$
\left(\frac{M-k a}{M}\right) s+\left(\frac{k(a+b)-M}{M}\right) t=1,
$$

where $0<\frac{M-k a}{M} \leq 1$ and $0 \leq \frac{k(a+b)-M}{M}<1$.

Using the fact that $\frac{1}{M} \in G$, let $h, h^{\prime}$ be homomorphisms from $R$ to $R$ (and then from $M_{N}(R)$ to $\left.M_{N}(R)\right)$ with $h_{*}=\frac{M-k a}{M}$ and $h_{*}^{\prime}=\frac{k(a+b)-M}{M}$. Then

$$
\rho_{R}\left(h_{*}[q]\right)+\rho_{R}\left(h_{*}^{\prime}\left[q^{\prime}\right]\right)=1 .
$$

From this, it can be seen that $h(q)$ and $h^{\prime}\left(q^{\prime}\right)$ can be taken to belong to $R$. From $\left[h^{\prime}\left(q^{\prime}\right)\right]=\left[1_{R}-h(q)\right], h^{\prime}$ can be chosen to satisfy $h^{\prime}\left(q^{\prime}\right)=1_{R}-h(q)$ and then $h(\gamma(A)) \subset h(q) R h(q)$ and $h^{\prime}(\mu(A)) \subset\left(1_{R}-h(q)\right) R\left(1_{R}-h(q)\right)$. Let $\Phi=h \circ \gamma+h^{\prime} \circ \mu$. Then $\Phi$ is a unital, completely positive contraction from $A$ to $R$. Since both $\mu$ and $\gamma$ were chosen to be $\frac{1}{2} \delta$-multiplicative on $\mathcal{G}$ and $h, h^{\prime}$ are norm reducing, $\Phi$ is $\delta$ multiplicative on $\mathcal{G}$. Furthermore, by the first paragraph of the proof,

$$
\rho_{R}\left(\Phi_{\sharp}(p)\right)=\rho_{R}\left(h_{*}\left(\gamma_{\sharp}(p)\right)\right)+\rho_{R}\left(h_{*}^{\prime}\left(\mu_{\sharp}(p)\right)\right),
$$

so $\rho_{R}\left(\Phi_{\sharp}(p)\right)=\rho_{A}([p])$ for each $p \in \mathcal{P}$.

\section{Antisymmetries}

The main result of the paper now follows as in Theorem 6.12 of [6], without the complication of non-zero $K_{1}$-groups.

Theorem 13. Let $A$ be a real $C^{*}$-algebra with complexification $A \otimes \mathbb{C}$ isomorphic to a UHF-algebra with infinitely many $2 \times 2$ matrix factors and let $R$ be the real $\mathrm{UHF}$-algebra of the same type as $A \otimes \mathbb{C}$. Then $A$ is isomorphic to $R$.

Proof. Let $\mathcal{F} \subset A$ be finite, let $\epsilon>0$ and let $(\mathcal{P}, \mathcal{G}, \delta)$ be the $K^{C R}$-triple given by Proposition 8. By Proposition 12 there exists a completely positive unital contraction $\Phi: A \rightarrow R$ such that $\Phi$ is $\delta$-multiplicative on $\mathcal{G}$ and $\rho_{R}\left(\Phi_{\sharp}(p)\right)=\rho_{A}([p])$ for each $p \in \mathcal{P}$. By Theorem 7.4 of $\left[22\right.$ (noting that $1 \in B_{n}$ for each $n$ ), there exists a unital *-homomorphism $\gamma: R \rightarrow A$, which (because the relevant groups are all isomorphic to $G \subset \mathbb{Q}$ ) induces an isomorphism from $K^{C R}(R)$ onto $K^{C R}(A)$. Let $\left\{R_{k}: k \in \mathbb{N}\right\}$ be a sequence of full real matrix algebras converging to $R$ and let $\left\{\eta_{k}: R \rightarrow R_{k}: k \in \mathbb{N}\right\}$ be a sequence of completely positive contractions converging to the identity map in the point norm topology. (For $r=a \otimes b$, with $a \in R_{k}$ and $b$ in the relative commutant of $R_{k}$, let $\eta_{k}(r)=$ Trace $(b) a$.) Then $\gamma \eta_{k} \Phi: A \rightarrow A$ is a completely positive contraction. We can then choose $k$ large enough to obtain $\gamma_{*}\left(\eta_{k} \Phi\right)_{\sharp}(p)=[p]$ for all $p \in \mathcal{P}$. It follows from Proposition 8 that there exists a unitary $u \in A$ so that $\beta=A d(u) \circ \gamma$ satisfies $\left\|\beta \eta_{k} \Phi(a)-a\right\|<\epsilon$ for all $a \in \mathcal{F}$ and hence $\mathcal{F} \subset_{\epsilon} \beta\left(R_{k}\right)$, which is isomorphic to $R_{k}=M_{n_{k}}(\mathbb{R})$ for some $n_{k}$. As in the 
complex case, described in Theorem 2.2 of [5], this establishes that $A$ is approximately finite dimensional. From the known classification of approximately finite dimensional real structures in $A \otimes \mathbb{C}$, described in [7, 8, 20], 21] $A$ is therefore isomorphic to $R$.

\section{Appendix: The universal COefFicient theorem FOR $\sigma$-UNITAL $C^{*}$-ALGEBRAS}

\section{Jeffrey L. Boersema And N. Christopher Phillips}

In 17, the universal coefficient theorem is stated for all pairs of complex $C^{*}$ algebras $(A, B)$ such that $A$ is in the bootstrap category of separable $C^{*}$-algebras $\mathcal{N}$ and $B$ is any $\sigma$-unital $C^{*}$-algebra. However, it has been known for several years that the proof in 17] fails in the case that $B$ is $\sigma$-unital but not separable. The problem is that if the $K$-theory of $B$ is not countably generated, the construction of the geometric free resolution in [18] produces algebras which are no longer $\sigma$-unital. In that case, the construction of the geometric injective resolution of [17] will also involve non- $\sigma$-unital $C^{*}$-algebras.

The same issue arises in the universal coefficient theorem for real $C^{*}$-algebras in 3]. In that case, the theorem is stated with the hypothesis that $B$ is separable in order to avoid these problems.

However, in both the real and complex cases, it is highly desired to have a Universal Coefficient Theorem for $\sigma$-unital $C^{*}$-algebras. In the classification of tracially $\mathrm{AF} C^{*}$-algebras (see Section 4 of [6], Section 5 of [16], and Section 4 above) the UCT is applied in a setting where $B$ is a non-separable $C^{*}$-algebra of the form $\prod B_{i} / \sum B_{i}$.

The purpose of the present note is to prove a result which will restore the validity of the universal coefficient theorem under the more general assumption that $B$ is $\sigma$-unital, in both the real and complex cases. Theorems 14 and 15 below are improvements of Theorem 3.2 of [17] and Proposition 4.6 of [3], respectively. The key point is that for any $C^{*}$-algebra $B$ we can now arrange a homomorphism where the target algebra $D$ is $\sigma$-unital and the induced map on $K$-theory is surjective. With these theorems, the published proofs of both Theorem 1.17 of [17] and Theorem 1.1 of [3] carry through for $\sigma$-unital $B$.

Theorem 14. Let $B$ be a complex $C^{*}$-algebra. Then there exists a $\sigma$-unital $C^{*}$ algebra $D$ such that $K_{*}(D)$ are injective groups and a $C^{*}$-algebra map $f: S B \rightarrow D$ such that the induced map

$$
K_{*}(S B) \stackrel{f_{*}}{\longrightarrow} K_{*}(D)
$$

is a monomorphism.

Theorem 15. Let $B$ be a real $C^{*}$-algebra. Then there exists a $\sigma$-unital $C^{*}$-algebra $D$ such that $K^{C R T}(D)$ is an injective CRT-module and a $C^{*}$-algebra map $f: S \mathfrak{S} B \rightarrow$ $D$ such that the induced map

$$
K^{C R T}(S \mathfrak{S} B) \stackrel{f_{*}}{\longrightarrow} K^{C R T}(D)
$$

is a monomorphism.

Proof. Let $B$ be a complex $C^{*}$-algebra which, as in the proof of [17], we may assume is unital. Let $r: F \rightarrow B \otimes \mathcal{K}$ be the first step in the geometric free resolution as in 
[18. Note that $F$ may not be $\sigma$-unital. The unitized homomorphism

$$
r^{+}: F^{+} \rightarrow(B \otimes \mathcal{K})^{+}
$$

is still part of a geometric resolution in the sense that $\left(r^{+}\right)_{*}$ is surjective and $K_{*}\left(F^{+}\right)$ consists of free groups.

Following the proof of Theorem 3.2 of [17, using $r^{+}$instead of $r$ produces an algebra $D$ whose $K$-theory is injective and a homomorphism $S(B \otimes \mathcal{K})^{+} \rightarrow D$ whose induced map on $K$-theory is monomorphic. Composing with the homomorphism $S B \rightarrow S(B \otimes \mathcal{K})^{+}$based on a rank 1 projection of $\mathcal{K}$ gives the desired $f$.

The algebra $D$ is realized through a series of constructions involving mapping cones and tensor products with separable $C^{*}$-algebras. Since the starting point for these constructions is the homomorphism $r^{+}$involving unital $C^{*}$-algebras, the lemma below guarantees that $D$ is $\sigma$-unital. This completes the proof of Theorem 14. The proof of Theorem 15 is achieved by a similar modification of the proof of Proposition 4.6 of [3].

Lemma 16. Let $f: A \rightarrow B$ be a homomorphism of $C^{*}$-algebras and let

$$
C f=\{(\phi, a) \mid \phi \in C([0,1], B), a \in A, \phi(0)=0, \phi(1)=f(a)\}
$$

be the mapping cone. If $A$ and $B$ are $\sigma$-unital, then so is $C f$.

Proof. Let $\left\{a_{n}\right\}_{n \in \mathbb{N}}$ and let $\left\{b_{n}\right\}_{n \in \mathbb{N}}$ be approximate identities for $A$ and $B$, respectively. Then $\left\{\left(\phi_{n}, a_{n}\right)\right\}_{n \in \mathbb{N}}$ is an approximate identity for $C f$, where $\phi_{n} \in$ $C([0,1], B)$ is defined by

$$
\phi_{n}(t)= \begin{cases}n t b_{i}, & 0 \leq t \leq 1 / n \\ b_{i}, & 1 / n \leq t \leq 1-1 / n, \\ n(1-t) b_{i}+(n(t-1)+1) f\left(a_{i}\right), & 1-1 / n \leq t \leq 1\end{cases}
$$

\section{REFERENCES}

[1] Bruce Blackadar, Symmetries of the CAR algebra, Ann. Math. 131 (1990), 589-623. MR.1053492 (91i:46084)

[2] Jeffrey L. Boersema, Real $C^{*}$-algebras, united $K$-theory, and the Künneth formula, $K$-Theory 26 (2002), 345-402. MR.1935138 (2004b:46072)

[3] Jeffrey L. Boersema, Real $C^{*}$-algebras, united $K K$-theory, and the Universal Coefficient Theorem, K-Theory 33 (2004), 107-149. MR.2131747 (2006d:46090)

[4] A.K. Bousfield, A classification of K-local spectra, J. Pure Appl. Algebra 66 (1990), 121-163. MR1075335 (92d:55003)

[5] Ola Bratteli, Inductive limits of finite dimensional $C^{*}$-algebras, Trans. Amer. Math. Soc. 171 (1972), 195-234. MR0312282 (47:844)

[6] M.Dadarlat and S. Eilers, On the classification of nuclear $C^{*}$-algebras, Proc. London Math. Soc. (3) 85 (2002), 168-210. MR1901373 (2003d:19006)

[7] T. Giordano, A classification of approximately finite real $C^{*}$-algebras, J. Reine Angew Math. 385 (1988), 161-194. MR931219 (89h:46078)

[8] K.R. Goodearl and D. Handelman, Classification of ring and $C^{*}$-algebra direct limits of finitedimensional semisimple real algebras, Mem. Amer. Math. Soc. 69 (1987), no. 372. MR.904013 (88k:46067)

[9] Beatrice J. Hewitt, On the homotopical classification of $K O$-module spectra, Ph.D. thesis, University of Illinois at Chicago, 1996, ProQuest LLC. MR2695203

[10] Kjeld Knudsen Jensen and Klaus Thomsen, Elements of $K K$-theory, Birkhäuser, Boston, Basel, Berlin, 1991. MR 1124848 (94b:19008) 
[11] G. G. Kasparov, Hilbert $C^{*}$-modules: Theorems of Stinespring and Voiculescu, J. Operator Theory 4 (1980), 133-150. MR587371 (82b:46074)

[12] H. Lin, Tracially AF $C^{*}$-algebras, Trans. Amer. Math. Soc. 353 (2001), 693-722. MR.1804513 (2001j:46089)

[13] H. Lin, The tracial topological rank of $C^{*}$-algebras, Proc. London Math. Soc. 83 (2001), 199-234. MR.1829565 (2002e:46063)

[14] H. Lin, An introduction to the classification of amenable $C^{*}$-algebras, World Scientific, Singapore, 2001. MR1884366 (2002k:46141)

[15] H. Lin, Stable approximate unitary equivalence of homomorphisms, J. Operator Theory 47 (2002), 343-378. MR1911851 (2003c:46082)

[16] H. Lin, Classification of simple $C^{*}$-algebras of tracial topological rank zero, Duke Math. J. 125 (2004), 91-119. MR2097358(2005i:46064)

[17] J. Rosenberg and C. Schochet, The Künneth theorem and the universal coefficient theorem for Kasparov's generalized $K$-functor, Duke Math. J. 55 (1987), 431-474. MR894590 (88i:46091)

[18] C. Schochet, Topological methods for $C^{*}$-algebras II: Geometric resolutions and the Künneth Formula, Pacific J. of Math. 98 (1982), 443-458. MR650021 (84g:46105b)

[19] G. Skandalis, Une notion de nucléarité en $K$-théorie (d'après J. Cuntz), K-Theory 1 (1988), 549-573. MR953916 (90b:46131)

[20] P.J. Stacey, Involutory *-antiautomorphisms in a direct limit of matrix algebras, J. London Math Soc. (2) 30 (1984), 486-500. MR810958 (88f:46118)

[21] P.J. Stacey, Real structure in direct limits of finite dimensional $C^{*}$-algebras, J. London Math. Soc. (2) 35 (1987), 339-352. MR881522 (88g:46083)

[22] P.J. Stacey, Stability of involutory *-antiautomorphisms in UHF-algebras, J. Operator Theory 24 (1990), 57-74. MR.1086544 (92g:46077)

[23] P.J. Stacey, Real structure in unital separable simple $C^{*}$-algebras with tracial rank zero and with a unique tracial state, New York J. Math. 12 (2006), 1-5. MR 2259241 (2007h:46071)

[24] J. Boersema, Real $C^{*}$-algebras, united $K K$-theory, and the universal coefficient theorem, $K$-Theory 33 (2004), 107-149. MR:2131747 (2006d:46090)

Department of Mathematics, La Trobe University, Victoria 3086, Australia

E-mail address: P.Stacey@latrobe.edu.au

Department of Mathematics, Seattle University, Seattle, Washington 98122

E-mail address: boersema@seattleu.edu

Department of Mathematics, University of Oregon, Eugene, Oregon 97403

E-mail address: ncp@sdarkwing.uoregon.edu 